\title{
Comparação Entre Técnicas de Busca Meta-Heurísticas para a Otimização de um MRAC
}

\author{
Igor S. Castro* Fadul F. Rodor* Fernando H. D. Guaracy** \\ Moisés M. Gonçalves * \\ * Instituto de Ciências Tecnológicas, Universidade Federal de Itajubá - \\ Campus Itabira, MG (e-mail: igor887@hotmail.com; \\ fadulrodor@unifei.edu.br;moisesgonc@hotmail.com). \\ ** Instituto de Engenharia de Sistemas e Tecnologias da Informação, \\ Universidade Federal de Itajubá, MG (e-mail: \\ fernandoh@unifei.edu.br).
}

\begin{abstract}
In this paper, metaheuristic search algorithms were used in order to search optimal values for a MRAC (Model-Reference Adaptive Controller), applied to the position control of a servo motor. This MRAC was designed using Lyapunov stability. Each search technique was executed several times in MATLAB ${ }^{\circledR}$, and after satisfactory results were obtained, the controller was implemented in Simulink $^{\circledR}$, and after that, in the real plant. The efficiency of the method was verified with data obtained in real experiments.

Resumo: Neste artigo, algoritmos de busca meta-heurísticas foram utilizados com o intuito de buscar valores ótimos dos parâmetros de um MRAC (Model-Reference Adaptive Controller), aplicado no controle de posição de um servo motor. O $M R A C$ foi embasado na teoria de estabilidade de Lyapunov. Para a obtenção dos valores dos ganhos de adaptação, executouse diversas vezes os algoritmos de busca no MATLAB $^{\circledR}$, e após atingir resultados satisfatórios, o controle foi implementado no Simulink ${ }^{\circledR}$. A eficiência do método foi verificada através da obtenção de dados coletados em experimentos reais.
\end{abstract}

Keywords: Model Reference Adaptive Control; Metaheuristic Search; Position Control; PIV.

Palavras-chaves: Controle Adaptativo por Modelo de Referência; Busca Meta-heurística; Controle de Posição; PIV.

\section{INTRODUÇÃO}

Para muitas aplicações industriais, a teoria de controle clássico oferece resultados satisfatórios. Um exemplo disso é o controle PID e suas variações que, mesmo quando na ausência informações exatas sobre o modelo matemático, é capaz de fornecer resultados satisfatórios (Ogata, 2010).

Contudo, nem sempre estruturas de controle clássicas são eficientes. Há casos em que o sistema apresenta distúrbios não modelados que são inerentes a seu funcionamento, em que há comportamentos não lineares, ou em que há parâmetros desconhecidos e/ou variantes no tempo. Nessas situações, não é incomum que técnicas clássicas de controle falhem em atingir o nível de desempenho desejado, e é neste contexto que a teoria de controle adaptativo se mostra relevante.

Embora ainda não haja um consenso sobre o que configura um controlador adaptativo, uma definição para tal seria "um controlador com parâmetros variáveis, e um mecanismo de ajuste para estes parâmetro" (Åström and Wittenmark, 1994). Atualmente, com a disponibilidade de microprocessadores poderosos por um preço acessível, muitos avanços foram feitos na área de controle adaptativo não linear (Slotine and Li, 1991). Um tipo específico de controlador adaptativo é o MRAC ou "Model-Reference Adaptive Controller", no qual as especificações de desempenho do sistema são dadas por um modelo de referência, e o mecanismo de adaptação busca minimizar o erro entre tal modelo e a saída real da planta, alterando os parâmetros da estrutura de controle no processo.

Cuong et al. (2013) ilustra, através de simulações, a eficiência deste tipo de controlador. Ao utilizá-lo com uma estrutura PV (proporcional-velocidade), e aplicá-lo a um sistema de segunda ordem cuja dinâmica varia com o tempo, é possível ver como a adaptação responde às mudanças da planta, encontrando novos valores para os ganhos do controlador. Em Nair et al. (2016) empregouse o $M R A C$ no controle de uma planta cujos parâmetros variam continuamente, comparando-o com outro tipo de controlador adaptativo e atestando sua eficiência.

Um dos importantes parâmetros de projeto de um $M R A C$ é o ganho de adaptação. Contudo, a complexidade da análise dinâmica de sistemas adaptativos torna a escolha de um valor para tal ganho uma tarefa pouco intuitiva. Uma possível solução para este problema é a escolha destes ganhos baseada na otimização de algum critério. Nounou and Passino (2000) emprega uma estrutura de auto-tuning 
para isso, de forma que os valores destes ganhos variem de acordo com uma lei de sintonia que garante a estabilidade do sistema. Já Dey et al. (2018) propõe outro tipo de sistema com ganhos de adaptação variáveis, no qual o sinal usado para se determinar tais ganhos é o erro de estimação dos parâmetros de controle. Tal método garante a robustez da performance do controle mesmo com a presença de incertezas.

Neste trabalho, contudo, procurou-se avaliar a obtenção de valores para os ganhos de adaptação do $M R A C$ através de dois métodos de busca meta-heurística, a "Otimização por Enxame de Partículas Quânticas" e por "Algoritmos Genéticos". Após o desenvolvimento e diversas execuções dos algoritmos de busca, os melhores valores obtidos para os parâmetros do $M R A C$ foram então implementados em uma simulação do sistema de controle no Simulink ${ }^{\circledR}$.

O artigo está organizado da seguinte maneira: a Seção 2 fará uma introdução da teoria do $M R A C$. Na Seção 3 serão abordadas as técnicas de busca meta-heurísticas utilizadas, enquanto a Seção 4 apresenta a metodologia proposta para este problema específico (o controle de posição de um servo motor). A Seção 5 contém os resultados obtidos experimentalmente, e, finalmente, a Seção 6 oferece uma conclusão acerca do trabalho.

\section{MODEL-REFERENCE ADAPTIVE CONTROL - $M R A C$}

Uma importante classe de controladores adaptativos é a dos MRACs. Este tipo de controlador expressa o desempenho desejado em termos de uma função objetivo, que dita a resposta "ideal" a um sinal de entrada. O sistema é dividido em dois laços de realimentação: Uma realimentação comum, composta pelo controlador e a planta real, e uma outra realimentação que altera os ganhos do controlador. A Figura 1 demonstra o diagrama de blocos deste tipo de controlador.

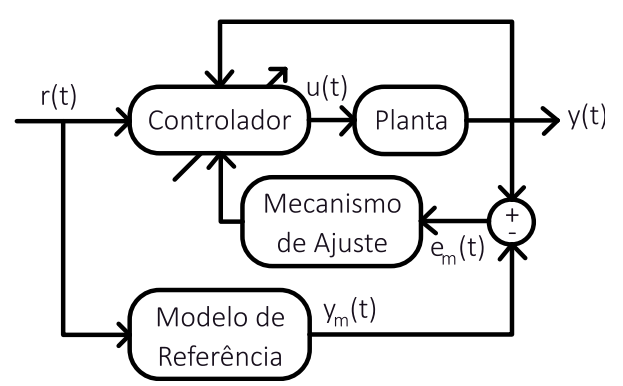

Figura 1. Diagrama de Blocos de um $M R A C$.

Na Figura 1, $r(t)$ representa o sinal de referência, $u(t)$ o sinal de controle, $y(t)$ a saída da planta, $y_{m}(t)$ a saída do modelo de referência, e $e_{m}(t)$ o erro entre a saída da planta e o modelo de referência.

Há duas principais formas de se determinar os mecanismos de adaptação: a partir de métodos baseados no gradiente, ou usando-se a teoria da estabilidade de Lyapunov, que será abordada neste artigo.

\subsection{Teoria da Estabilidade de Lyapunov}

Para o projeto de um $M R A C$ usando-se a teoria da estabilidade de Lyapunov (Åström and Wittenmark, 1994; Slotine and Li, 1991), uma abordagem por análise em espaço de estados se torna mais adequada. Primeiramente, considera-se um sistema linear em malha fechada descrito por

$$
\dot{x}=A(\theta) x+B(\theta) r
$$

em que $x$ corresponde ao vetor de estados da planta, $A$ é uma matriz quadrada que determina a relação entre os estados, $B$ é o vetor que relaciona a referência aos estados, e $r$ é o sinal de referência. Neste caso, as matrizes $A$ e $B$ são dadas em função de $\theta$, que é um vetor composto por todos os parâmetros ajustáveis do controlador.

Em seguida, determina-se a representação em espaço de estados do modelo de referência, que é dada por

$$
\dot{x}_{m}=A_{m} x_{m}+B_{m} r .
$$

Assim, considera-se que existe um grupo de parâmetros $\theta^{0}$ que resulta em

$$
\begin{aligned}
& A\left(\theta^{0}\right) \approx A_{m} \\
& B\left(\theta^{0}\right) \approx B_{m} .
\end{aligned}
$$

Para se definir o mecanismo de adaptação que resulta nas considerações descritas por (3), define-se o erro $e_{m}$,

$$
e_{m}=x-x_{m}
$$

de forma que sua derivada pode ser obtida subtraindo (2) de (1), resultando em

$$
\dot{e}_{m}=A(\theta) x+B(\theta) r-A_{m} x_{m}-B_{m} r .
$$

Ao se adicionar e subtrair $A_{m} x$ do lado direito da equação, a mesma pode ser reorganizada na forma

$$
\dot{e}_{m}=A_{m} e_{m}+\left(A(\theta)-A_{m}\right) x+\left(B(\theta)-B_{m}\right) r .
$$

Outra forma de se expressar (6) é reorganizando-se os termos que multiplicam o vetor de estados $x$ e a referência $r$. Para isso, considerando-se as condições (3), introduz-se a matriz $\Psi$, que é expressa através do produto

$$
\Psi\left(\theta-\theta^{0}\right)=\left(A(\theta)-A\left(\theta^{0}\right)\right) x+\left(B(\theta)-B\left(\theta^{0}\right)\right) r
$$

na qual o vetor $\theta$ contém os parâmetros adaptáveis da estrutura de controle, e o vetor $\theta^{0}$ contém os valores destes parâmetros que satisfazem (3). Assim, $\left(\theta-\theta^{0}\right)$ é o vetor de erros entre os parâmetros do controlador, e os parâmetros ideais que levariam o comportamento do sistema real a se aproximar do modelo de referência. Desta forma, (6) se torna

$$
\dot{e}_{m}=A_{m} e_{m}+\Psi\left(\theta-\theta^{0}\right) .
$$

De posse destas equações, é necessário agora encontrar uma função de Lyapunov que permita a obtenção do mecanismo de adaptação. Para isso, introduz-se a função $V\left(e_{m}, \theta\right)$, dada por

$$
V\left(e_{m}, \theta\right)=\frac{1}{2}\left(e_{m}^{T} P e_{m}+\left(\theta-\theta^{0}\right)^{T} \Gamma^{-1}\left(\theta-\theta^{0}\right)\right)
$$

em que $P$ é uma matriz simétrica positiva definida e $\Gamma$ é a matriz positiva definida de ganhos de adaptação. Para verificar se $V\left(e_{m}, \theta\right)$ é uma função de Lyapunov, ela deve ser positiva definida (o que é garantido por sua natureza quadrática), e sua derivada deve ser negativa semidefinida. 
Para verificar essa segunda condição, deriva-se (9) em relação ao tempo, obtendo-se assim

$$
\begin{gathered}
\dot{V}=-\frac{1}{2} e_{m}^{T} Q e_{m}+\left(\theta-\theta^{0}\right)^{T} \Psi^{T} P e_{m}+\left(\theta-\theta^{0}\right)^{T} \Gamma^{-1} \dot{\theta} \\
=-\frac{1}{2} e_{m}^{T} Q e_{m}+\left(\theta-\theta^{0}\right)^{T}\left(\Gamma^{-1} \dot{\theta}+\Psi^{T} P e_{m}\right)
\end{gathered}
$$

em que a matriz $Q$ é positiva definida que satisfaz

$$
A_{m}^{T} P+P A_{m}=-Q .
$$

Assim, é suficiente definir a lei de ajuste de parâmetros como

$$
\dot{\theta}=-\Gamma \Psi^{T} P e_{m}
$$

de forma que (10) se torna

$$
\dot{V}=-\frac{1}{2} e_{m}^{T} Q e_{m}
$$

que é negativa semidefinida. Isto confirma que a função $V\left(e_{m}, \theta\right)$ é uma função de Lyapunov, e a lei de ajuste de parâmetros escolhida resulta em um sistema estável em malha fechada (considerando-se que o modelo de referência é estável e o sinal de entrada é limitado). Contudo, é importante ressaltar que, para garantir que $\dot{V}$ se aproxime de zero a medida que o tempo se aproxima do infinito, é necessário adicionalmente que $\ddot{V}$ seja limitada, de acordo com o lema de Barbalat (Aström and Wittenmark, 1994).

\section{TÉCNICAS DE BUSCA META-HEURÍSTICA}

Na seção anterior, foi possível ver que um dos parâmetros de projeto do $M R A C$ presente no mecanismo de adaptação é o ganho de adaptação, para o qual não há uma regra única e objetiva que garanta um bom desempenho devido à natureza complexa de sua influência no sistema.

Neste contexto, técnicas de busca meta-heurística se tornam relevantes, uma vez que, partindo de princípios relativamente simples, são capazes de encontrar soluções que atendam determinados critérios para problemas de grande complexidade matemática. As duas técnicas de otimização empregadas neste trabalho, sendo algorítimos baseados em busca populacional, possuem uma estrutura principal semelhante, contudo cada uma delas tem características únicas que as tornam mais eficientes em diferentes tipos de problemas.

A estrutura geral dos métodos empregados é ilustrada de forma simplificada na Figura 2.

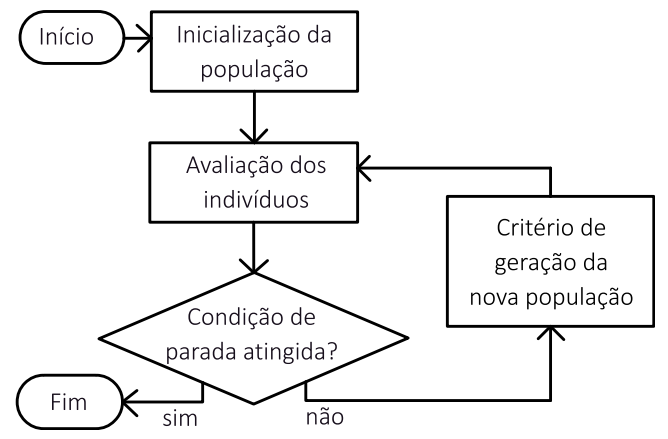

Figura 2. Diagrama de Blocos de um Algoritmo de Busca Meta-heurístico Baseado em Busca Populacional

\subsection{Genetic Algorithms - GAs}

Os GAs, ou "Algoritmos Genéticos", estão entre as mais bem conhecidas técnicas de busca meta-heurística. Desenvolvido originalmente por Holland (1975), um GA busca inspiração na teoria da evolução das espécies para otimizar uma função específica.

A execução de um $G A$ necessita, inicialmente, que uma população seja gerada. Esta população é dada por "indivíduos" que usualmente são representados por palavras binárias. O número de bits por indivíduo depende do tamanho e precisão do intervalo de busca, já que tais indivíduos refletem pontos no espaço de possibilidades.

Uma vez que os indivíduos são gerados, cada um deles é então avaliado de acordo com uma função fitness, que determina o quão "adaptados" eles estão. Adota-se, para problemas de minimização, a consideração de que um menor valor de fitness corresponde a uma maior qualidade do indivíduo. A determinação da função fitness é um dos problemas principais na elaboração de um algoritmo genético, pois ela dita quais características serão mais desejadas na população.

Depois que os indivíduos são avaliados, é necessário selecionar quais deles irão compor a próxima geração. O método adotado neste trabalho é o de "torneio". Com este método, dois membros da população são escolhidos aleatoriamente, e então, o melhor indivíduo passa para a próxima geração. O processo é repetido até que a nova geração tenha a mesma quantidade de indivíduos que a população original.

Uma vez selecionados, os indivíduos da nova geração são separados em pares, e podem ou não realizar um "cruzamento" (ou crossover). A ocorrência deste depende da chamada "taxa de cruzamento", um dos principais parâmetros a ser determinado no projeto de um $G A$, já que ela previne convergências prematuras e garante a variabilidade das gerações.

Caso o cruzamento entre um par ocorra, em um primeiro momento escolhe-se um ponto aleatório nas palavras binárias do par, separando-os em duas partes cada. Em seguida, estas partes são trocadas entre os indivíduos.

Por fim, após possibilitar que os indivíduos da nova geração realizem o cruzamento, a população resultante passa pelo processo de "mutação". Nesta etapa, cada bit dos indivíduos pode "mutar", o que representa sofrer a operação lógica "not". A probabilidade de cada bit sofrer uma mutação está relacionada à "taxa de mutação".

Após a mutação, uma nova geração é gerada com sucesso, e todo o processo pode ser realizado novamente. É frequente também a adoção do "elitismo", no qual o melhor indivíduo da geração anterior passa para a próxima sem alterações.

A condição de parada irá depender do problema específico, mas não é incomum impor um limite de gerações. Um aprofundamento sobre este algoritmo meta-heurístico pode ser encontrado no trabalho de Reeves and Rowe (2002).

\subsection{Quantum-behaved Particle Swarm Optimization - QPSO}

Outra técnica de otimização meta-heurística é o PSO, ou "Otimização por Enxame de Partículas", proposto origi- 
nalmente por Kennedy and Eberhart (1995). Este tipo de algoritmo simula o movimento das partículas de busca com base no comportamento de um bando de pássaros ou um cardume de peixes. É um método que se aproveita da "inteligência de grupo" das partículas para explorar o espaço de possibilidades.

Um aprimoramento deste algorítimo é o QPSO, ou "Quantum-behaved Particle Swarm Optimization", explorado pela primeira vez por Sun et al. (2004). Este método meta-heurístico aproveita-se das características estocásticas do comportamento de uma partícula quântica, de forma que é capaz de explorar de forma eficiente grandes intervalos de busca.

O QPSO tem início ao se gerar o conjunto de partículas, cada uma delas representando uma posição no espaço de soluções. Essas partículas são então avaliadas de acordo com a função fitness.

A equação que determina a nova posição de cada partícula em uma nova geração depende da função de probabilidade escolhida. No caso deste artigo, a estrutura escolhida é dada por

$$
S_{n, d}^{i+1}=p_{n, d}^{i} \pm \beta\left|S_{n, d}^{i}-C_{d}^{i}\right| \ln \frac{1}{{ }_{1} U_{n, d}^{i}}
$$

em que $S$ representa a posição da partícula, $i$ a geração, $d$ a dimensão e $n$ o indivíduo da população. $\beta$ é a constante de convergência, $C$ o valor da média entre as melhores posições já atingidas por cada partícula, e ${ }_{1} U$ é um valor real aleatório entre 0 e 1.

$\mathrm{O}$ valor de $p$ é definido por

$$
p_{n, d}^{i}={ }_{2} U_{n, d}^{i} M_{n, d}^{i}+\left(1-{ }_{2} U_{n, d}^{i}\right) G_{n}^{j}
$$

em que ${ }_{2} U$ é outro valor real aleatório entre 0 e $1, M$ é a melhor posição já alcançada pela partícula, e $G$ é a melhor posição já alcançada dentre todas as partículas.

Para o algoritmo utilizado na realização deste trabalho, uma versão do QPSO com controle de diversidade foi adotada. Nesta versão, a "diversidade" das partículas (uma medida da distância média entre elas) é calculada a cada iteração. Caso a diversidade esteja abaixo de um valor limite, a constante de convergência $\beta$ é então alterada para um valor que causa divergência na posição das partículas. Quando o valor de diversidade se torna novamente maior que o limite, a constante de convergência retorna ao seu valor original.

Uma vez que as partículas se movem, elas são reavaliadas e o processo se inicia novamente. Sun and $\mathrm{Wu}$ (2011) oferece em seu trabalho um aprofundamento neste tipo de algoritmo, abordando também suas diversas variações.

\section{OTIMIZAÇÃO DOS GANHOS DE ADAPTAÇÃO}

Esta seção abordará as características individuais tanto do problema de controle, quanto das técnicas empregadas para solucioná-lo.

\subsection{Controle de Posição de um Servo Motor}

A planta a ser controlada pelo $M R A C$ consiste em um carro acoplado a uma trilha por meio de engrenagens. O servo motor que o movimenta tem tensão nominal de $\pm 6 \mathrm{~V}$, e um encoder também acoplado ao carro fornece a velocidade do mesmo. O sistema real pode ser observado na Figura 3.

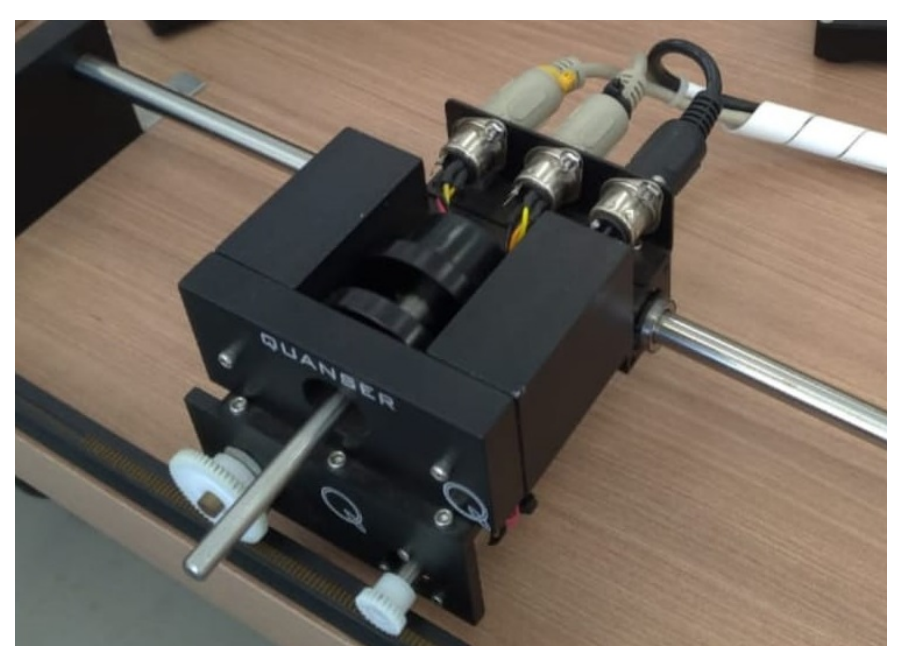

Figura 3. Sistema de posição

A modelagem da resposta de velocidade do motor pode ser encontrada no manual do fabricante Quanser (2012). Nesta modelagem, um polo relacionado à indutância do motor é ignorado, uma vez que sua constante de tempo é desprezível se comparada com a constante de tempo da parte mecânica da planta. Assim, a resposta do servo é aproximada por um sistema de primeira ordem descrito por

$$
\frac{V_{c}(s)}{V_{m}(s)}=\frac{K_{g}}{\tau s+1}
$$

em que o ganho $K_{g}$ tem valor $0,143[\mathrm{~m} / \mathrm{s} / \mathrm{V}]$ e a constante de tempo $\tau$ é equivalente a $0,0584[\mathrm{~s}] . V_{c}$ é a velocidade do carro em metros por segundo, e $V_{m}$ é a tensão de entrada no motor em Volts. Estas constantes foram obtidas a partir dos valores nominais da planta, sendo razoável assumir que esses valores não são exatos (desgaste natural do modelo, diferenças nos processos de fabricação, etc).

Como esta função de transferência fornece a velocidade em função da tensão aplicada ao motor, para encontrar a resposta em posição, acrescenta-se um elemento integrador, de forma que a equação se torna

$$
\frac{X_{c}(s)}{V_{m}(s)}=\frac{0,143}{s(0,0584 s+1)}
$$

em que $X_{c}$ é a posição do carro em metros.

Para o controle desta planta, optou-se pela estrutura de um PIV com compensação feedforward. Este tipo de controle foi escolhido pois, apesar de a planta já possuir um polo na origem (o que, em malha fechada, garantiria o rastreamento de referências do tipo degrau em regime permanente), há distúrbios relacionados aos atritos estático e dinâmico no motor. Tais distúrbios não são integrados pela própria planta, o que faz necessário que outro elemento integrativo seja introduzido. O diagrama de blocos da estrutura de controle é ilustrado na Figura 4. 


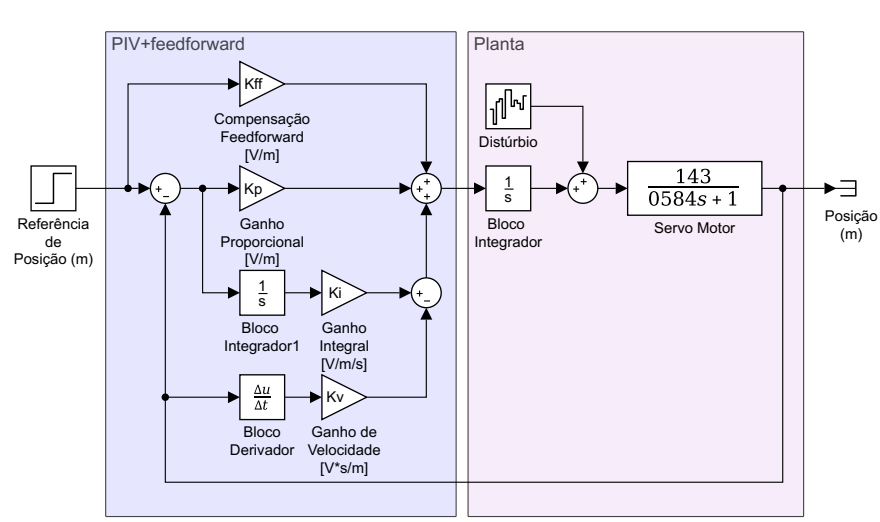

Figura 4. Estutura de Controle de um PIV com Feedforward

O PIV é composto por um parâmetro $K_{P}$ relacionado ao erro $E(s)$, um parâmetro $K_{I}$ associado à integral deste erro, e um parâmetro $K_{V}$ ligado à derivada da saída $Y(s)$. A compensação feedforward, presente para rejeição de distúrbios, acrescenta um ganho $K_{F F}$ relacionado ao valor da referência $R(s)$. O sinal de controle deste PIV foi saturado em $\pm 8 \mathrm{~V}$ para se evitar danos ao motor.

Assim, é possível então determinar a função de transferência do sistema em malha fechada, dada por

$$
\frac{Y(s)}{R(s)}=\frac{s\left(K_{P}+K_{F F}\right) K_{g}+K_{g} K_{I}}{s^{3} \tau+s^{2}\left(1+K_{g} K_{V}\right)+s K_{g} K_{P}+K_{g} K_{I}} .
$$

\subsection{Funções de Adaptação do MRAC-Lyapunov}

Como o desenvolvimento do $M R A C$-Lyapunov fora, neste artigo, realizado a partir de uma abordagem em espaço de estados, é necessário então determinar-se quais estados serão considerados na representação do sistema. A partir de (18), que dita o comportamento do sistema em malha fechada, é possível então se obter a equação diferencial

$$
\begin{gathered}
\dddot{y}(t) \tau+\ddot{y}(t)\left(1+K_{g} K_{V}\right)+\dot{y}(t) K_{g} K_{P}+y(t) K_{g} K_{I}= \\
\dot{r}(t)\left(K_{P}+K_{F F}\right) K g+r(t) K_{g} K_{I}
\end{gathered}
$$

e após integrá-la dos dois lados da equação, obtém-se

$$
\begin{gathered}
\ddot{y}(t) \tau+\dot{y}(t)\left(1+K_{g} K_{V}\right)+y(t) K_{g} K_{P}+K_{g} K_{I} \int y(t) d t= \\
r(t)\left(K_{P}+K_{F F}\right) K g+K_{g} K_{I} \int r(t) d t
\end{gathered}
$$

Dessa forma, escolhe-se os estados como

$$
\begin{gathered}
x_{1}=\int r(t) d t-\int y(t) d t=\int e(t) d t \\
x_{2}=y(t) \\
x_{3}=\dot{y}(t)
\end{gathered}
$$

o que resulta na representação em espaço de estados

$$
\begin{gathered}
\dot{x}(t)=A(\theta) x(t)+B(\theta) r(t) \\
A(\theta)=\left[\begin{array}{ccc}
0 & -1 & 0 \\
0 & 0 & 1 \\
\frac{K_{g}}{\tau} K_{I} & -\frac{K_{g}}{\tau} K_{P}-\frac{\left(1+K_{V} K_{g}\right)}{\tau}
\end{array}\right] \\
B(\theta)=\left[\begin{array}{c}
1 \\
0 \\
\frac{K g}{\tau}\left(K_{F F}+K_{P}\right)
\end{array}\right]
\end{gathered}
$$

Assim, considerando que existe um vetor de parâmetros ideais $\theta^{0}$ que resulta na expressão dada por (3), é possível recorrer à (7) para determinar-se a matriz $\Psi$. Assim, obtém-se

$$
\begin{gathered}
\theta-\theta^{0}=\left[\begin{array}{c}
K_{V}-K_{V}^{0} \\
K_{P}-K_{P}^{0} \\
K_{F F}-K_{F F}^{0}
\end{array}\right] \\
\Psi=\frac{K_{g}}{\tau}\left[\begin{array}{ccc}
0 & 0 & 0 \\
0 & 0 & 0 \\
r(t) & e(t) & -\dot{y}(t)
\end{array}\right] .
\end{gathered}
$$

Uma vez determinada a matriz $\Psi$, recorre-se à (12) para se determinar a taxa de variação dos parâmetros do controlador. Sendo

$$
\begin{gathered}
\Gamma=\left[\begin{array}{ccc}
\gamma_{1} & 0 & 0 \\
0 & \gamma_{2} & 0 \\
0 & 0 & \gamma_{3}
\end{array}\right] \\
e_{m}=\left[\begin{array}{c}
x_{1}-x_{1 m} \\
x_{2}-x_{2 m} \\
x_{3}-x_{3 m}
\end{array}\right]=\left[\begin{array}{c}
y_{m}(t) d t-\int y(t) d t \\
y(t)-y_{m}(t) \\
\dot{y}(t)-\dot{y}_{m}(t)
\end{array}\right] .
\end{gathered}
$$

O modelo de referência selecionado é uma função de segunda ordem com frequência natural $\omega_{n}=11,277 \mathrm{e}$ fator de amortecimento $\zeta=0,591$ acrescida de um zero e um polo que se cancelam. Tal modelo é descrito pelas equações de estado

$$
\begin{gathered}
x_{m 1}=\int_{x_{m 2}=y_{m}(t)} r(t) d t y_{m}(t) d t \\
x_{m 3}=\dot{y}_{m}(t) . \\
\dot{x}(t)=A_{m}(\theta) x_{m}(t)+B_{m}(\theta) r(t) \\
B_{m}(\theta)=\left[\begin{array}{c}
1 \\
0 \\
127,2
\end{array}\right] \\
A_{m}(\theta)=\left[\begin{array}{ccc}
0 & -1 & 0 \\
0 & 0 & 1 \\
3179,4 & -460,5 & -38,3
\end{array}\right]
\end{gathered}
$$

\subsection{A Função Fitness}

A seleção da função fitness é uma das decisões mais importantes na implementação de um algoritmo de otimização. É a partir dela que o algoritmo meta-heurístico avalia a qualidade das partículas em seu espaço de busca, e pequenas variações em tal função podem acarretar grandes alterações nos resultados. Para este problema em específico, optou-se por ponderar dois parâmetros principais nesta função fitness. O primeiro deles foi o erro quadrático médio (EQM) ponderado, e o segundo deles, o esforço de controle. 
O erro quadrático médio ponderado pode ser obtido, neste caso, ao se fazer a média ponderada do quadrado do erro $e_{m}$. Assim, sua expressão é

$$
E Q M=\frac{1}{N} \sum_{k=1}^{N} Z(k)\left(e_{m}(k)\right)^{2}
$$

em que $N$ é o número de amostras, $Z_{j}$ é a $k$-ésima amostra do vetor de pesos, e $e_{m}(k)$ é a $k$-ésima amostra do erro entre a saída da planta e modelo de referência.

$\mathrm{O}$ vetor de pesos $Z(k)$ foi definido de forma empírica através da comparação dos resultados de diversas simulações, e é dado por

$$
Z(k)=14,22 \exp \left(k \times 8,533 \times 10^{-4}\right)
$$

A natureza exponencial do vetor de pesos $Z(k)$ faz com que qualquer erro $e_{m}(k)$ tenha pouca relevância no início da simulação, mas passe a ser penalizado com mais rigor a medida que o tempo passa. Essa característica foi adotada pois, em um $M R A C$, é comum que haja um erro maior durante os primeiros instantes de funcionamento (enquanto está em período de adaptação). Contudo, a medida em que o tempo passa, é desejável que o erro entre o sistema controlado e o modelo de referência seja mínimo, e por isso há a necessidade de uma penalização maior.

Já o esforço de controle (EC) foi calculado a partir de

$$
E C=\sum_{k=1}^{N}|u(k)| T_{s} \max (|u|)^{1,4}
$$

na qual $u(k)$ é o $k$-ésimo elemento do sinal de controle, $T_{s}$ é o período de amostragem e $\max (|u|)$ é o maior valor assumido pelo sinal de controle.

Ao se computar o EC desta forma, planejou-se penalizar sistemas que resultassem em sinais de controle muito elevados. Assim, de posse das definições de EQM e EC, o valor da função objetivo pôde ser definido como

$$
F=\log (E Q M+E C) \text {. }
$$

Vale ressaltar que, nos cálculos realizados para a obtenção dos valores de EQM e EC, buscou-se garantir que os valores resultantes apresentassem a mesma ordem de grandeza.

\section{$4 \cdot 4$ Simulações}

Uma vez definido a estrutura do $M R A C$ a ser testado, os métodos de otimização, e a função objetivo, foram então realizadas diversas simulações. Cada algorítimo de busca foi executado 30 vezes em uma máquina que usava Windows 8 (64 bits) como sistema operacional, possuía 8Gb de memória RAM, e processador Intel i7-6500.

Todos os algoritmos utilizaram 100 indivíduos por geração, e executaram por 250 gerações. O espaço de busca para os valores das constantes de adaptação foi de 0 a $1 \times 10^{6}$.

Para o GA, fora adotada uma taxa de mutação de 2,5\%, taxa de cruzamento de $55 \%$, e torneio com 2 indivíduos. Já para o QPSO, a constante de convergência adotada se iniciava em 1,25, mas decaía linearmente com as iterações até atingir 0,5 .

Os ganhos de adaptação relacionados a $K_{V}, K_{P}$ e $K_{F F}$ são, respectivamente, $\gamma_{1}, \gamma_{2}$ e $\gamma_{3}$. Optou-se por manter o valor do ganho integral estático para reduzir o período de adaptação, que se mostrou demasiado alto com 4 parâmetros variáveis. Os resultados obtidos estão expostos na Tabela 1.

Tabela 1. Resultado das Simulações para o MRAC-Lyapunov

\begin{tabular}{ccc}
\hline Parâmetro & GA & QPSO \\
\hline \hline $\operatorname{Best}(\mathrm{F})$ & 2,9796 & 2,9795 \\
\hline $\operatorname{Mean}(\mathrm{F})$ & 2,9805 & 2,9796 \\
\hline $\operatorname{Var}(\mathrm{F})\left[\times 10^{-7}\right]$ & 9,0791 & $7.55 \times 10^{-4}$ \\
\hline $\operatorname{Best}\left(\gamma_{1}\right)\left[\times 10^{3}\right]$ & 1,7177 & 1,7162 \\
\hline $\operatorname{Mean}\left(\gamma_{1}\right)\left[\times 10^{3}\right]$ & 1,7974 & 1,7183 \\
\hline $\operatorname{Var}\left(\gamma_{1}\right)\left[\times 10^{4}\right]$ & 1,2421 & $3.2494 \times 10^{-3}$ \\
\hline $\operatorname{Best}\left(\gamma_{2}\right)\left[\times 10^{3}\right]$ & 8,5187 & 8,5081 \\
\hline $\operatorname{Mean}\left(\gamma_{2}\right)\left[\times 10^{3}\right]$ & 8,3304 & 8,5165 \\
\hline $\operatorname{Var}\left(\gamma_{2}\right)\left[\times 10^{5}\right]$ & 2,7119 & $3,9086 \times 10^{-3}$ \\
\hline $\operatorname{Best}\left(\gamma_{3}\right)\left[\times 10^{5}\right]$ & 1,5123 & 1,5124 \\
\hline $\operatorname{Mean}\left(\gamma_{3}\right)\left[\times 10^{5}\right]$ & 1,5253 & 1,5119 \\
\hline $\operatorname{Var}\left(\gamma_{3}\right)\left[\times 10^{6}\right]$ & 9,5838 & $1.6688 \times 10^{-2}$ \\
\hline
\end{tabular}

Em que Best, Mean e Var representam, respectivamente, o melhor valor, o valor médio e a variância encontrados para cada variável.

A evolução média do melhor valor de fitness por geração pode ser analisado a partir da Figura 5.

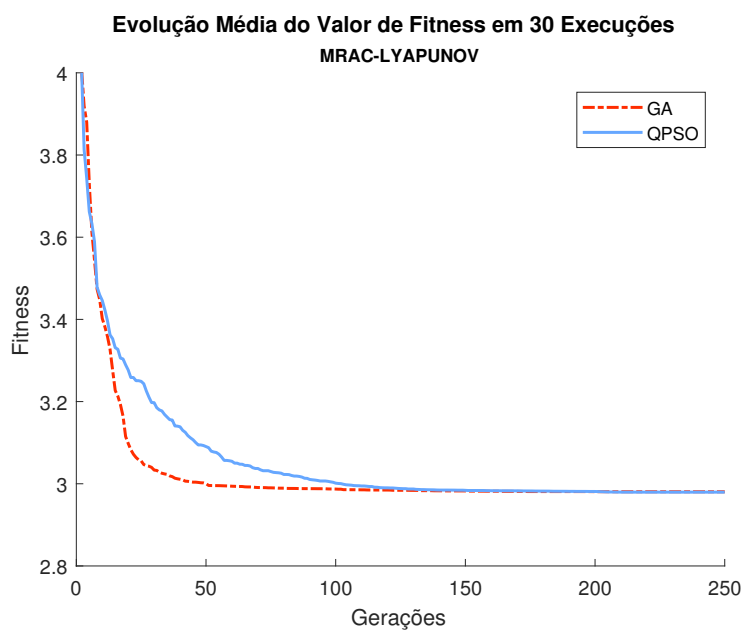

Figura 5. Evolução do Melhor Valor de Fitness para os 2 Métodos de Otimização

Nota-se que o $G A$ convergiu mais rapidamente, contudo, o QPSO foi capaz de encontrar melhores valores ao final das 250 gerações.

Utilizando-se os valores obtidos pelo $Q P S O$, realizou-se uma simulação para verificar o comportamento da estratégia de controle em análise. A resposta das simulações do $M R A C$ está ilustrada na Figura 6. 


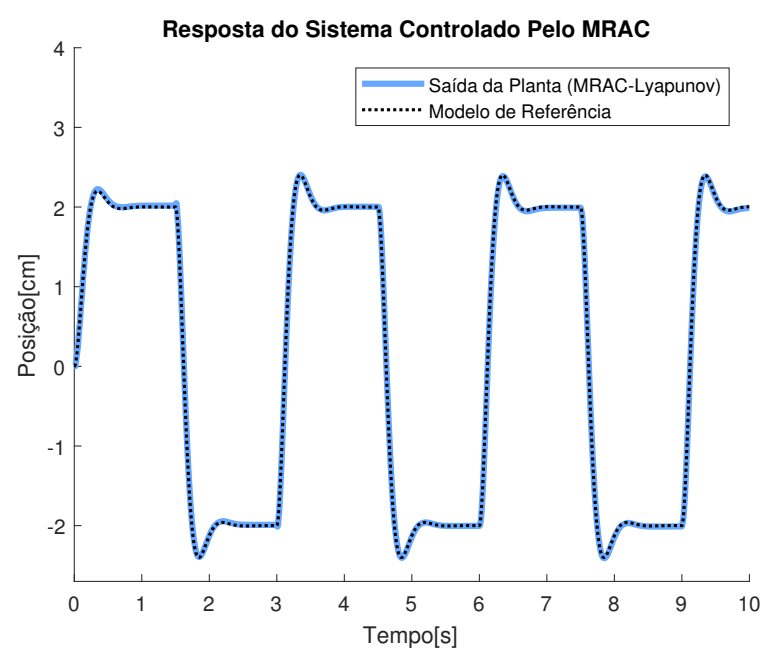

Figura 6. Simulação do $M R A C$ com Ganhos Encontrados - Saída

É possível ver, a princípio, que o $M R A C$ segue o modelo de referência em um curto período de tempo. O sinal de comando do motor pode ser observado na Figura 7.

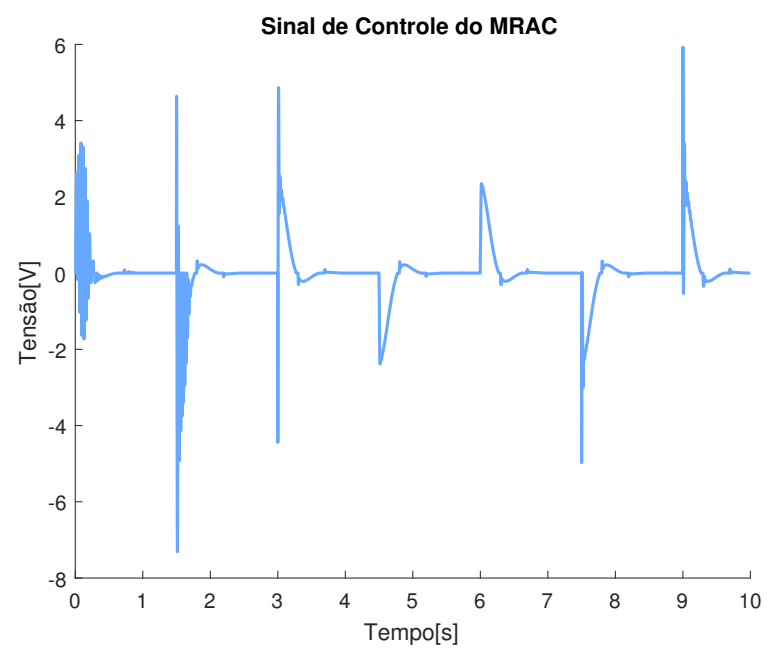

Figura 7. Simulação do $M R A C$ com Ganhos Encontrados - Sinal de Controle

Ao analisar o sinal de comando, percebe-se um comportamento mais "agressivo" da atuação, principalmente nos estágios iniciais, uma vez que há grandes picos e uma natureza oscilatória.

\section{RESULTADOS EXPERIMENTAIS}

O MRAC com ganhos de adaptação encontrados foi então implementado na planta real. A Figura 8 apresenta a saída da planta.

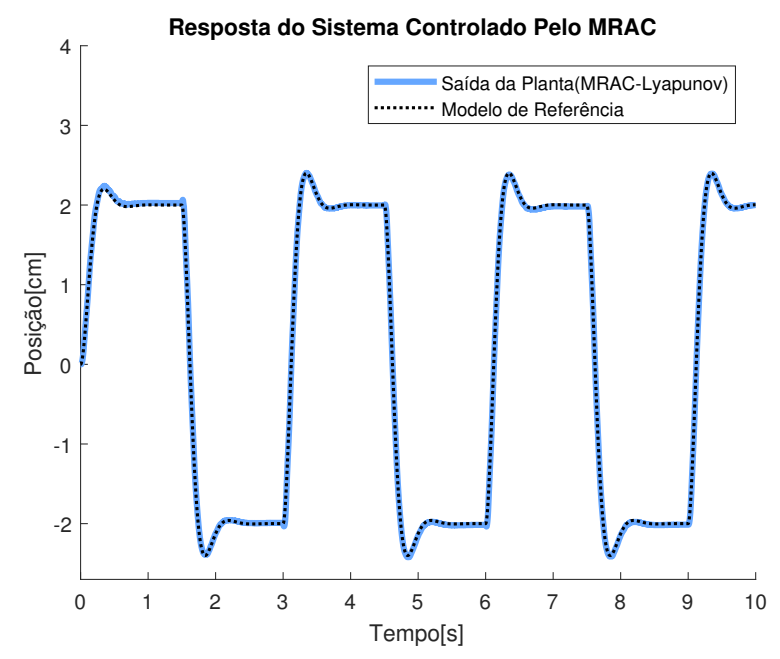

Figura 8. Dados Experimentais do $M R A C$ com Ganhos Ótimos - Saída

Verifica-se que o comportamento da planta real se aproximou do comportamento simulado, uma vez que passou a seguir o modelo de referência rapidamente. O sinal de comando resultante pode ser observado na Figura 9.

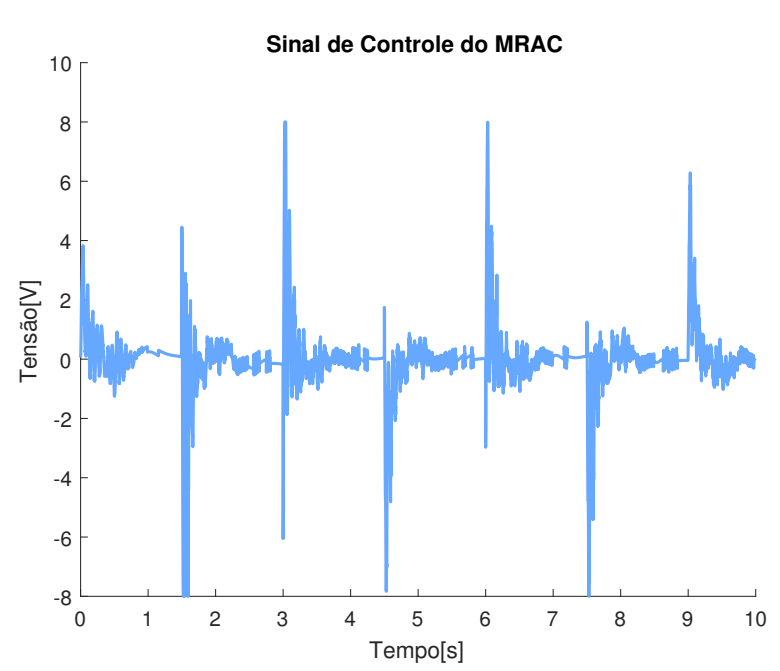

Figura 9. Dados Experimentais do $M R A C$ com Ganhos Encontrados - Sinal de Controle

Os sinais de comando obtidos experimentalmente também apresentaram comportamento semelhante ao simulado, contudo o controle tornou-se consideravelmente mais "agressivo", possivelmente para compensar incertezas e distúrbios não modelados.

A Figura 10 ilustra a evolução dos ganhos proporcional e feedforward do controlador. Nota-se que os ganhos tendem a se estabilizar em torno de uma região de operação, mas ainda sim apresentam variações consideráveis durante a execução do algoritmo de controle. 


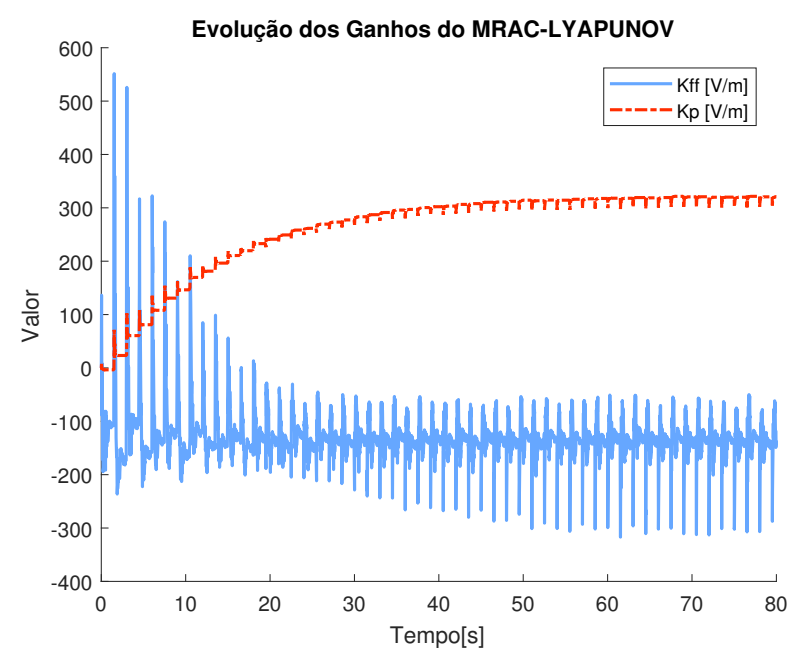

Figura 10. Dados Experimentais do MRAC-Lyapunov com Ganhos Encontrados - Evolução de Kp e $K_{f f}$

A Figura 11 ilustra a evolução do ganho de velocidade do $M R A C$.

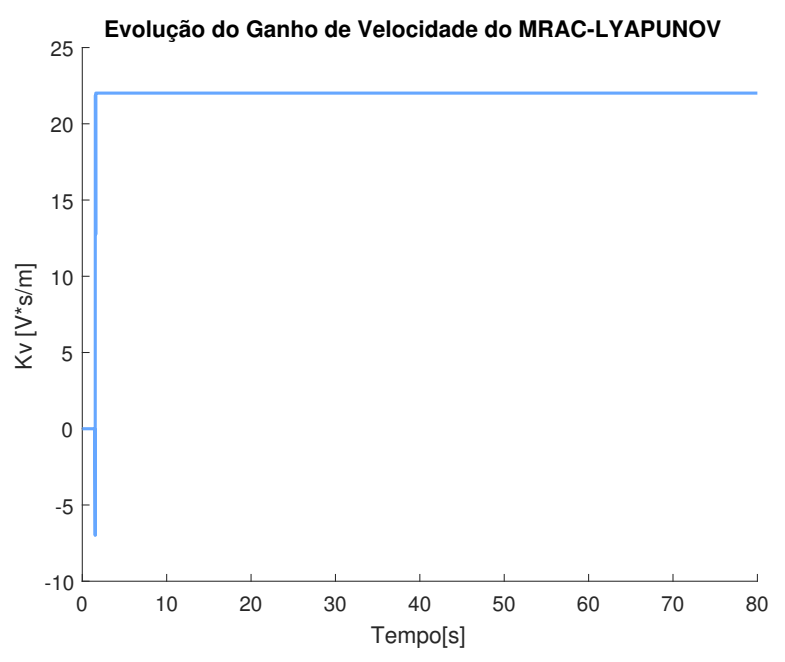

Figura 11. Dados Experimentais do MRAC-Lyapunov com Ganhos Ótimos - Evolução de Kv

Percebe-se que o valor deste ganho atinge seu valor final rapidamente, possivelmente pois, para a variação deste ganho específico, a adaptação fora realizada somente quando o erro entre saída da planta e modelo de referência era maior que $2 \%$.

\section{CONCLUSÕES}

Neste artigo, técnicas de busca meta-heurística foram utilizadas na otimização dos ganhos de adaptação de um $M R A C$ baseado em uma estrutura PIV com ganho feedforward.

Todos os algoritmos de otimização conseguiram encontrar valores para os ganhos de adaptação capazes de produzir resultados aceitáveis. Contudo, diferenças entre tais métodos puderam ser observadas. Apesar de o GA e o $Q P S O$ apresentarem resultados semelhantes, o último utiliza uma estrutura consideravelmente mais simples, já que possui menos parâmetros de configuração.

O MRAC baseado na teoria de Lyapunov obteve resultados satisfatórios tanto nas simulações quanto na aplicação real do controlador, exibindo uma adaptação rápida e minimizando o erro entre saída e modelo de referência em um curto período de tempo.

Em trabalhos futuros pretende-se analisar novas funções de custo, assim como a introdução de distúrbios e ruídos no processo. É também possível o estudo da aplicação deste método em plantas não lineares ou com atraso de transporte.

\section{REFERÊNCIAS}

Cuong, N.D., Lanh, N., and Huyen, D.V. (2013). Design of mras-based adaptive control systems. In International Conference on Control, Automation and Information Sciences, 79-84. IEEE.

Dey, R., Jain, S.K., and Padhy, P.K. (2018). Robust flexible adaptation gain based crm for guaranteed transient performance. Transactions of the Institute of Measurement and Control.

Holland, J.H. (1975). Adaptation in Natural and Artificial Systems: An Introductory Analysis with Applications to Biology, Control, and Artificial Intelligence. University of Michigan Press.

Kennedy, J. and Eberhart, R. (1995). Particle swarm optimization. In International Conference on Neural Networks. IEEE.

Nair, A.P., Narayanasamy, S., and Lalithambika, V.R. (2016). Lyapunov based pd/pid in model reference adaptive control for satellite launch vehicle systems. Aerospace Science and Technology, 51, 70-77.

Nounou, H.N. and Passino, K.M. (2000). Stable autotuning of the adaptation gain for direct adaptive control. In American Control Conference, 2154-2158. IEEE.

Ogata, K. (2010). Modern Control Engineering. Prentice Hal, 5 edition.

Quanser (2012). Linear Servo Base Unit Workbook.

Reeves, C.R. and Rowe, J.E. (2002). Genetic Algorithms - Principles and Perspectives: A Guide to GA Theory. Operations Research Computer Science Interfaces Series. Springer, 1 edition.

Slotine, J.J.E. and Li, W. (1991). Applied Nonlinear Control. Pearson, 1 edition.

Sun, J., Feng, B., and Xu, W. (2004). Particle swarm optimization with particles having quantum behavior. In Congress on Evolutionary Computation, 325-331. IEEE.

Sun, J. and Wu, C.H.L.X.J. (2011). Particle Swarm Optimisation: Classical and Quantum Perspectives. CRC Press, 1 edition.

Åström, K.A. and Wittenmark, B. (1994). Adaptive Control. Prentice Hall, 2 edition. 